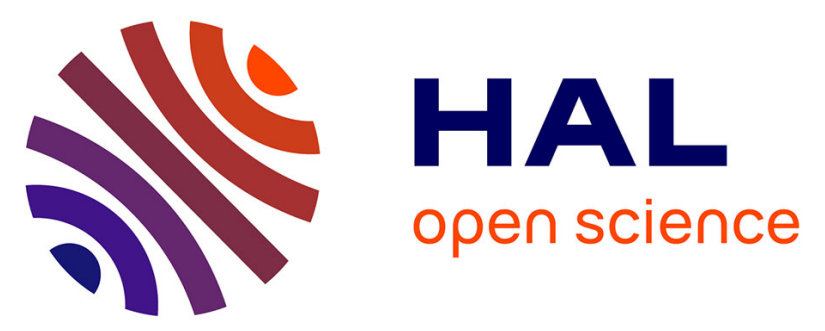

\title{
X-ray and Radio Timing of PSR B1821-24
}

Paul.S. Ray, Michael T. Wolff, Paul Demorest, Ismaël Cognard, Donald C.

Baker, Kent S. Wood

\section{To cite this version:}

Paul.S. Ray, Michael T. Wolff, Paul Demorest, Ismaël Cognard, Donald C. Baker, et al.. X-ray and Radio Timing of PSR B1821-24. 40 YEARS OF PULSARS: Millisecond Pulsars, Magnetars and More, Aug 2007, Montreal, Canada. pp.157-159, 10.1063/1.2900134 . insu-02926354

\section{HAL Id: insu-02926354 https://hal-insu.archives-ouvertes.fr/insu-02926354}

Submitted on 31 Aug 2020

HAL is a multi-disciplinary open access archive for the deposit and dissemination of scientific research documents, whether they are published or not. The documents may come from teaching and research institutions in France or abroad, or from public or private research centers.
L'archive ouverte pluridisciplinaire HAL, est destinée au dépôt et à la diffusion de documents scientifiques de niveau recherche, publiés ou non, émanant des établissements d'enseignement et de recherche français ou étrangers, des laboratoires publics ou privés. 


\title{
X-ray and Radio Timing of PSR B1821-24
}

\author{
Paul S. Ray*, Michael T. Wolff*, Paul Demorest ${ }^{\dagger}$, Ismaël Cognard**, Donald C. \\ Backer $^{\ddagger}$ and Kent S. Wood*
}

\author{
* Space Science Division, Naval Research Laboratory, Washington, DC 20375-5352 \\ ${ }^{\dagger}$ National Radio Astronomy Observatory, Charlottesville, VA 22903 \\ ** LPCE-CNRS, F-45071 Orleans, France \\ ${ }^{\ddagger}$ Astronomy Department, University of California, Berkeley, CA 94720
}

\begin{abstract}
We report on long term timing observations of the $3.05 \mathrm{~ms}$ pulsar PSR B1821-24, made with the Proportional Counter Array (PCA) on NASA's Rossi X-ray Timing Explorer (RXTE), which have an absolute pulse time of arrival accuracy of $<3.5 \mu \mathrm{s}$. We compare these observations to careful radio timing observations taken at Nançay Observatory and the Green Bank Telescope, which allow us to track the variable dispersion measure so that we can compensate for radio propagation effects. These data are the first high precision long term timing measurements of a millisecond pulsar in the X-ray band and demonstrate that timing of X-ray emitting MSPs in the X-ray band can be an important complement to radio timing programs.
\end{abstract}

Keywords: pulsars

PACS: $97.60 . \mathrm{Gb}$

\section{INTRODUCTION}

Rotation-powered millisecond pulsars (RMSPs) are most often discovered and studied at radio frequencies because they are bright, coherent, radio emitters and there are several large radio telescopes that devote significant observing time to their study. However, the X-ray emission from these pulsars is also of great interest for several reasons: (1) a much larger fraction of the spindown energy loss rate is radiated in X-ray compared to the radio band, (2) the X-rays are emitted via an incoherent emission mechanism which likely makes the X-ray profile much more stable and predictable than the radio profile, (3) comparison of the X-ray, $\gamma$-ray, and radio profiles can help constrain the emission sites within the magnetosphere, and (4) there appears to be a relationship between the non-thermal X-ray emission and the production of radio giant pulses. The launch of the GLAST observatory, expected in mid-2008, will bring a major new capability to study of these sources and will provide a new opportunity to study the phasing of emission components from radio to $\gamma$-rays.

Radio pulsar timing has yielded some of the most important results in modern astrophysics, from the unambiguous detection of the effects of gravitational radiation, to the precise masses of neutron stars. Timing also yields precise positions and proper motions for many pulsars, detections of planet-sized bodies orbiting pulsars, the gravitational effect of globular clusters, the noisy spindown of a superfluid interior imperfectly coupled to a normal matter crust, and several other General Relativistic effects in binary pulsars. In addition to these measurements directly related to the pulsar being observed, pul- sar timing is an excellent probe of the interstellar medium where effects such as dispersion, Faraday rotation, scattering, and both refractive and diffractive scintillation are routinely observed. These propagation effects yield rich information about the structure of the interstellar medium, but can become the limiting factor on the precision of radio pulsar timing. Timing RMSPs in the Xray band, effectively at infinite frequency, removes these propagation effects as well as difficult-to-calibrate instrumental polarization effects, and probably also the pulseto-pulse jitter and amplitude fluctuations that are a consequence of the coherent emission process responsible for the radio emission.

Precise long-term timing of an array of pulsars is sensitive to perturbations of the spacetime metric at the pulsar and at the Earth. A pulsar timing array can thus be a sensitive gravitational wave detector in the microHertz to nanoHertz regime. Current sensitivity levels achieved in timing the radio millisecond pulsars are approaching predictions for detecting the stochastic gravitational wave background and certain individual sources (Jenet et al. 2005, ApJ, 625, L123; Jenet, Creighton, \& Lommen 2005, ApJ, 627, L125). With radio pulsar timing hitting systematic limits that make achieving TOAs with accuracy much better than $100 \mathrm{~ns}$ difficult or impossible, a large area X-ray instrument may eventually be the best choice for this type of gravitational wave detector. Of course, this prospect can't fully be evaluated without considerably more effort to characterize the X-ray timing properties of RMSPs.

To date, X-ray timing observations of RMSPs have been one time observations, quite unlike the intensive, long-term campaigns that are common in the radio. The 
TABLE 1. Radio timing model used in this analysis PSR J1824-2452

RAJ 18:24:32.00790550 00.00001054

DECJ -24:52:10.8076448 00.0020416

F0 327.405606051749543900.0000000000233327

F1 -1.735361869603E-13 $01.123466933817 \mathrm{E}-18$

PEPOCH 53800.000000

DM 0.000356

EPHEM DE405

TZRMJD 53859.26176012789721

TZRFRQ 822.000

TZRSITE 1

JUMP $1-0.0001552310 .00000010$

JUMP_2 0.0013688610 .00000012

JUMP 30.0012931210 .00000061

observations described here, particularly when combined with earlier RXTE observations of these sources, will allow the first long-term X-ray timing of RMSPs and further the detailed understanding of the relationship of the radio to the $\mathrm{X}$-ray emission and their relative timing properties.

\section{PSR B1821 - 24}

The 3.05 ms pulsar PSR B1821-24, in the globular cluster M28, is the youngest known RMSP, and is the only RMSP to have exhibited a glitch (Cognard \& Backer 2004, ApJ, 612, L125) (in March of 2001). Its X-ray spectrum is a hard power law with a photon index of 1.3. Its X-ray light curve consists of two narrow peaks separated by $\sim 0.45$ in phase. The dispersion measure of the source is variable (Cognard \& Lestrade 1997, A\&A, 323,211 ), requiring careful, contemporaneous radio observations in order to make absolute $\mathrm{X}$-ray to radio phase comparisons. We have made regular observations of the source ( $>10$ times per month) at the Nançay Radiotelescope at three frequencies $(1400,1600, \& 2000 \mathrm{MHz})$, as well as monthly dual-frequency $(820 \mathrm{MHz}$ and 1430 $\mathrm{MHz}$ ) timing observations at the Green Bank Telescope to ensure that we have a precise radio ephemeris with accurate time-dependent DM throughout the X-ray monitoring.

The radio timing model we used for this work is based on a joint fit to Nançay and GBT monitoring of the source from February 2006 through April 2007 using the standard pulsar timing program TEMPO v11.005. We fit a model consisting of position, frequency, and frequency first derivative, as well as three time jumps to correct for the different alignments of the template profiles at the different frequencies and observatories (Table 1).

In addition, the dispersion measures used for the individual TOAs were derived from fits to the multifrequency GBT observations. The measured DMs are shown in Figure 1. For scale, note that an offset of 0.001

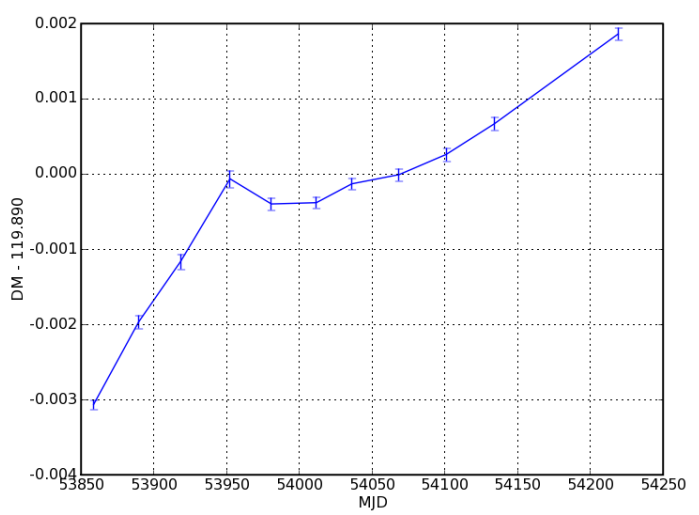

FIGURE 1. Dispersion measure variation vs. time determined from Green Bank Telescope monitoring at 1400 and 800 $\mathrm{MHz}$.

$\mathrm{pc} / \mathrm{cm}^{3}$ corresponds to an error of $6.5 \mu \mathrm{s}$ in the correction of the observed arrival time from $800 \mathrm{MHz}$ to infinite frequency.

In the timing model we neglect the proper motion of the source, which is fairly poorly determined despite the long-term timing as a result of a combination of timing noise (which is quite strong in this source compared to other millisecond pulsars) and DM variations that can not be compensated for in the earlier single frequency data. To calibrate the scale of the error we are making by assuming zero proper motion, we generated a set of fake TOAs using the "fake" plugin to the TEMPO2 pulsar timing package (Hobbs, Edwards, \& Manchester, 2006, MNRAS, 369, 655). Giving it a model with a proper motion of -0.417 mas/year in RA and -8.993 mas/year in DEC (corresponding to the best measured values from the Nançay long term timing), we generated monthly TOAs over an observing span of \pm 1000 days from the present. Allowing for a fit for the current position produces a model that results in errors of less than $1 \mu$ s over our $<2$ year data span.

\section{RXTE RESULTS}

For the current analysis we used all RXTE observations of PSR B1821-24 since the beginning of 2006, when our GBT radio monitoring campaign began. The RXTE data were processed using the standard HEAsoft FTOOLS (05Apr2005_V6.0). We selected data from good time intervals, defined as times where RXTE was pointed within 0.02 degrees of the source and the source was more than 5.0 degrees above the Earth limb. We tried many combinations of energy cuts and detector layer selection and found that the signal to noise was 


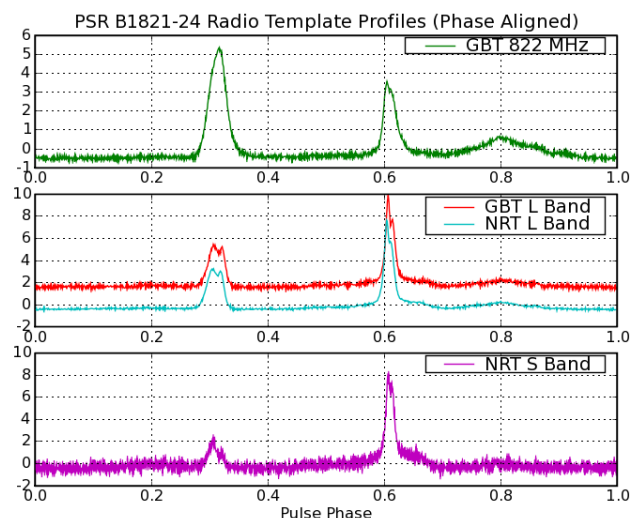

FIGURE 2. Phase-aligned radio template profiles.

maximized by using PCU Layers 1 and 2, and an energy cut of 3-15 keV (channels 8-37 in gain epoch 5), so these event filters were used for all processing. After the events were filtered, they were barycentered using the FTOOL faxbary using the position from the radio ephemeris above. The tool faxbary also applies the RXTE fine clock corrections resulting in event times in TDB believed to be accurate to better than $3.4 \mu$ s (Jahoda et al. 2006, ApJS, 163, 401).

Folding all of the RXTE data since 2006 (ObsIDs 92008-01-01-01, 92008-01-01-02, 92008-01-01-03, and 92804-01-01-01) according to the radio ephemeris with NO free parameters results in the pulse profile shown in Figure 3, with the phase-aligned $800 \mathrm{MHz}$ radio profile. The alignment is the same as that seen in previous measurements (e.g. Rots et al. 1998, ApJ, 501, 749), though observed here with much higher time resolution and signal to noise ratio.

We then generated folded X-ray profiles from each RXTE observation and computed pulse TOAs using the gaussian template determined above. These were fitted using TEMPO using only one parameter, an absolute phase jump that corresponds to the different template profile in X-ray vs. the radio, and the residuals are shown in Figure 4. The TOAs all agree with the radio ephemeris to well within the error estimate on the TOAs themselves. The RMS residual is only $0.6 \mu \mathrm{s}$.

\section{DISCUSSION}

These observations represent one of the first attempts to characterize the long term timing properties of a rotationpowered millisecond pulsar in the X-ray range. We find that the phase alignment between the radio and the X-ray profiles stays remarkably constant. In each 50 ks RXTE observation (using 3 PCUs) we are able to measure the

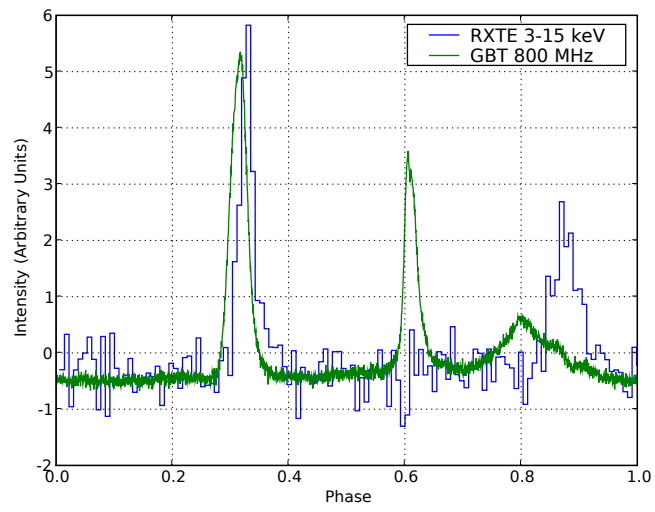

FIGURE 3. Phase aligned $3-15 \mathrm{keV} X$-ray (histogram) and $800 \mathrm{MHz}$ radio (solid line) profiles.

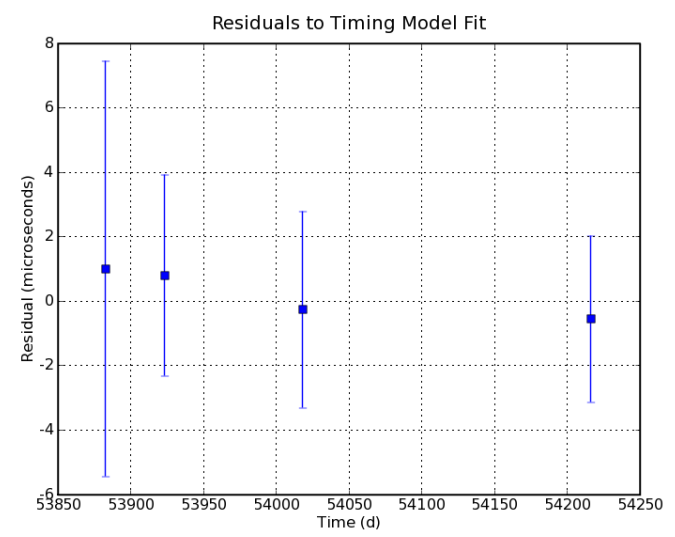

FIGURE 4. X-ray pulse TOA residuals to the radio timing model. The first point (with the larger error bar) is a 15ks public TOO observation, and the last three are our $50 \mathrm{ks}$ observations.

$\mathrm{X}$-ray TOAs to an accuracy of $\pm 3 \mu \mathrm{s}$. A future X-ray timing experiment that provided a larger effective area and lower background could significantly improve on this accuracy and would make timing of X-ray emitting RMSPs in the X-ray band competitive with the highest accuracy MSP timing in the radio band $(\sim 100 \mathrm{~ns}$ accuracy). This would be a powerful complement to radio timing programs since many of the systematic errors, particularly those resulting from propagation effects, are not present in the X-ray band.

\section{ACKNOWLEDGMENTS}

This work was partially supported by the NASA RXTE Guest Observer program and by NRL/ONR. 